\title{
ALL CLUSTER POINTS \\ OF COUNTABLE SETS IN SUPERCOMPACT SPACES ARE THE LIMITS OF NONTRIVIAL SEQUENCES
}

\author{
ZHONGQIANG YANG
}

(Communicated by Franklin D. Tall)

\begin{abstract}
A space is called supercompact if it has an open subbase such that every cover consisting of elements of the subbase has a subcover consisting of two elements. In this paper we prove that, in a continuous image of a closed $G_{\delta}$-set of a supercompact space, a point is a cluster point of a countable set if and only if it is the limit of a nontrivial sequence. As corollaries, we answer questions asked by J. van Mill et al.
\end{abstract}

In this paper we prove that, in a continuous image of a closed $G_{\delta}$-set of a supercompact space, any cluster point of a countable set is the limit of a nontrivial convergent sequence. As corollaries, we answer two questions asked by van Douwen and van Mill and by van Mill and Mills in [6] and [9], respectively.

Every space in the present paper is assumed to be Hausdorff, and the word "subbase" is assumed to mean "subbase for closed sets". For a space $X$ a family $\mathscr{F}$ of closed sets is called linked if $F \cap F^{\prime} \neq \varnothing$ for all $F, F^{\prime} \in \mathscr{F}$, and binary if every linked subfamily has nonempty intersection. A space is called supercompact if it has a binary subbase [7]. Many compact spaces, but not all, are supercompact. For example, all compact metric spaces are supercompact [5, 11]; all continuous images of compact ordered spaces are supercompact [4]. On the other hand, closed $G_{\delta}$-sets of supercompact spaces are neither supercompact in general [3] nor continuous images of supercompact spaces [10]. Moreover, Bell gave an example of a nonsupercompact dyadic space ( $=$ a continuous image of $2^{\kappa}$ ) [2].

In 1982 van Douwen and van Mill proved in [6] that, in a continuous image of a supercompact space, at least one cluster point of a countable set is the limit of a nontrivial convergent sequence in the whole space; at most countably many cluster points are not so. The result suggested to them the following question.

Question 1 [6]. Let $Y$ be a continuous image of a supercompact space (or just a supercompact space). If $K$ is a countable subset of $Y$, then is every cluster point of $K$ the limit of a nontrivial convergent sequence?

Applying the result mentioned above, van Mill and Mills proved in [9] that,

Received by the editors September 25, 1992 and, in revised form, January 4, 1993.

1991 Mathematics Subject Classification. Primary 54D30.

Key words and phrases. Supercompact, limit, sequence. 
under a set-theoretical hypothesis, every infinite continuous image of a closed $G_{\delta}$-set of a supercompact space contains a nontrivial convergent sequence. Then, they asked if the set-theoretical hypothesis may be dropped.

Question 2 [9]. If $Y$ is an infinite continuous image of a closed $G_{\delta}$-set of a supercompact space, then does $Y$ contain a nontrivial convergent sequence?

In this paper we prove the next theorem, which answers the above two questions affirmatively.

Theorem. Let $Y$ be a continuous image of a closed $G_{\delta}$-subset of a supercompact space and $K$ a countable subset of $Y$. Then every cluster point of $K$ is the limit of a nontrivial convergent sequence in $Y$.

To show the theorem, we first give some lemmas. The first two lemmas can be directly proved. Let $N$ be the set of all natural numbers.

Lemma 1. Let $f: X \rightarrow Y$ be a continuous mapping from a compact space $X$ onto a space $Y$ and $\left\{A_{n} \subset X: n \in N\right\}$ a decreasing sequence of closed sets of $X$. If $\bigcap_{n \in N} A_{n} \subset f^{-1}(y)$ for some $y \in Y$, then $f\left(a_{n}\right) \rightarrow y$ for any $a_{n} \in A_{n}$.

Now let $\mathscr{S}$ be a subbase (note that subbase means subbase for closed subsets) for a compact space $X$. Let $\mathscr{S}^{n}=\left\{\cap \mathscr{S}_{0}: \mathscr{S}_{0} \subset \mathscr{S}\right\}$. Then $\mathscr{S}^{n}$ is also a subbase. Furthermore, if $\mathscr{S}$ is binary, then $\mathscr{S}^{n}$ is also binary. Thus we assume throughout this paper that every subbase is closed with respect to arbitrary intersections. Now we fix a point $p \in X$. For $A \subset X$ let

$$
J(A)=\bigcap\{S \in \mathscr{S}: p \in S \text { and } S \cap A \neq \varnothing\} .
$$

If $A=\{a\}$, we write $J(a)$ instead of $J(\{a\})$.

Lemma 2. Let $\mathscr{S}$ be a subbase for a compact space $X, F$ a closed subset, and $U$ an open subset of $X$. If $F \subset U$, then there exist $S_{1}, S_{2}, \ldots, S_{n} \in \mathscr{S}$ such that $F \subset S_{1} \cup S_{2} \cup \cdots \cup S_{n} \subset U$. In particular, if a point $x \in U$, then there exist $S_{1}, S_{2}, \ldots, S_{n} \in \mathscr{S}$ such that $x \in S_{1} \cap S_{2} \cap \cdots \cap S_{n}$ and $x \in$ $\operatorname{int}\left(S_{1} \cup S_{2} \cup \cdots \cup S_{n}\right) \subset S_{1} \cup S_{2} \cup \cdots \cup S_{n} \subset U$.

Lemma 3. Let $A, B \subset X$. If $S \cap A \neq \varnothing$ implies $S \cap B \neq \varnothing$ for every $S \in \mathscr{S}$ with $p \in S$, then $p \in \bar{A}$ implies $p \in \bar{B}$. In particular, if $p \in \bar{A}$, then $J(A)=$ $\{p\}$.

Proof. Let $p \in \bar{A}$. If $p \notin \bar{B}$, then by Lemma 2 there exist $S_{1}, S_{2}, \ldots, S_{n} \in \mathscr{S}$ such that $p \in S_{1} \cap S_{2} \cap \cdots \cap S_{n}$ and

$$
p \in \operatorname{int}\left(S_{1} \cup S_{2} \cup \cdots \cup S_{n}\right) \subset S_{1} \cup S_{2} \cup \cdots \cup S_{n} \subset X \backslash \bar{B} .
$$

Since $p \in \bar{A}$, there exists $S_{i}$ such that $S_{i} \cap A \neq \varnothing$. Hence $S_{i} \cap B \neq \varnothing$, which contradicts (1). Now if $q \in J(A)$, then $S \cap A \neq \varnothing$ implies $q \in S$ for every $S \in \mathscr{S}$ with $S \ni p$. Thus we have $p \in \overline{\{q\}}=\{q\}$; that is, $J(A)=\{p\}$.

Lemma 4. Let $E, Z \subset X$ be closed sets and $C=\left\{c_{n}: n \in N\right\} \subset Z$ a countable set. If $p \in E \cap \bar{C}$ and $E \cap C=\varnothing$, then one of the following statements holds.

(A) There exists an increasing sequence $\left\{A_{n}: n \in N\right\}$ of subsets of $C$ such that $Z \cap J\left(A_{n}\right) \not \subset E$ for all $n \in N$ but $Z \cap \bigcap_{n \in N} J\left(A_{n}\right) \subset E$. 
(B) There exists a sequence $\left\{A_{n}: n \in N\right\}$ of subsets of $C$ such that $C=$ $\bigcup_{n \in N} A_{n}$ and $Z \cap J\left(A_{n}\right) \not \subset E$ but $Z \cap J\left(A_{n}\right) \cap J\left(A_{m}\right) \subset E$ for all $n, m \in N$, $n \neq m$.

Proof. Suppose that there exists no sequence of subsets of $C$ satisfying the conditions in (A). Then we construct $\left\{A_{n}: n \in N\right\}$ such that, for all $n \in N$ and $c \in C \backslash \bigcup_{i \leq n} A_{n}$,

$$
\begin{gathered}
Z \cap J\left(A_{n}\right) \not \subset E, \quad Z \cap J\left(A_{n}\right) \cap J(c) \subset E, \\
c_{k(n)} \in A_{n} \subset C \backslash \bigcup_{i<n} A_{i},
\end{gathered}
$$

where $k(n)$ is the least $k$ satisfying $c_{k} \in C \backslash \bigcup_{i<n} A_{i}$.

In fact, if $\left\{A_{i}: i<n\right\}$ have been defined satisfying the required conditions, then $\bigcup_{i<n} A_{i} \neq C$, since otherwise, $p \in \bar{A}_{i}$ for some $i<n$. Hence, Lemma 3 implies that $Z \cap J\left(A_{i}\right) \subset J\left(A_{i}\right)=\{p\} \subset E$, which contradicts the assumption. Since $C$ is countable, $c_{k(n)} \in C \backslash\left(E \cup \bigcup_{i<n} A_{i}\right)$, and (A) does not hold, there exists a maximal subset $A_{n}$ of $C \backslash \bigcup_{i<n} A_{i}$ such that $c_{k(n)} \in A_{n}$ and $Z \cap J\left(A_{n}\right) \not \subset$ $E$. Then for all $c \in C \backslash \bigcup_{i \leq n} A_{i}$ we have $Z \cap J\left(A_{n}\right) \cap J(c) \subset E$. The inductive definition is completed. It is clear that the sequence $\left\{A_{n}: n \in N\right\}$ satisfies the required conditions in (B).

Proof of the theorem. Suppose that $Y$ and $K \subset Y$ satisfy the conditions in Theorem and $y \in \bar{K} \backslash K$. Let $X$ be a supercompact space with a binary subbase $\mathscr{S}$ and $Z \subset X$ a closed $G_{\delta}$-set, and let $f: Z \rightarrow Y$ be a continuous mapping from $Z$ onto $Y$. Then there exist a countable set $C \subset Z$ and $p \in Z$ such that $f(C)=K$ and $p \in \bar{C} \cap f^{-1}(y)$. Clearly, $E=f^{-1}(y)$ and $Z$ and $C$ satisfy the requests in the last lemma. Hence there exists a sequence $\left\{A_{n}: n \in N\right\}$ of subsets of $C$ satisfying the conditions in (A) or (B). Choose $z_{n} \in Z \cap J\left(A_{n}\right) \backslash f^{-1}(y)$. Then $\left\{f\left(z_{n}\right): n \in N\right\}$ is a sequence in $Y$, and $f\left(z_{n}\right) \neq y$ for all $n \in N$. If (A) holds, then Lemma 1 implies $f\left(z_{n}\right) \rightarrow y$. If (B) holds, then by Lemma 3 we have that

$$
\begin{gathered}
p \in \overline{\left\{z_{n}: n \in N\right\},} \\
Z \cap J\left(z_{n}\right) \cap J\left(z_{m}\right) \subset f^{-1}(y)
\end{gathered}
$$

for all $n \neq m$. To complete the proof of the theorem, it suffices to show the following lemma.

Lemma 5. If $D=\left\{z_{n}: n \in N\right\} \subset Z \backslash f^{-1}(y)$ satisfies (2) and (3), then there exists a subsequence $\left\{z_{n_{k}}, k \in N\right\}$ of $\left\{z_{n}, n \in N\right\}$ such that $f\left(z_{n_{k}}\right) \rightarrow y$.

Proof. Since $Z$ is a $G_{\delta}$-set, we can put $Z=\bigcap\left\{U_{k}: k \in N\right\}$ for open subsets $U_{k}(k \in N)$ of $X$ with $U_{k+1} \subset U_{k}$. Then by Lemma 2 for every $k \in N$ there exist $S_{1}, S_{2}, \ldots, S_{m} \in \mathscr{S}$ such that

$$
p \in S_{1} \cap S_{2} \cap \cdots \cap S_{m}
$$

and

$$
p \in \operatorname{int}\left(S_{1} \cup S_{2} \cup \cdots \cup S_{m}\right) \subset S_{1} \cup S_{2} \cup \cdots \cup S_{m} \subset U_{k} .
$$

Since $p \in \bar{D}$, there exists $S_{i}$ such that $S_{i} \cap D$ is infinite. Thus $\left\{n: J\left(z_{n}\right) \subset U_{k}\right\}$ is infinite for $k \in N$. Therefore, we can inductively define $\left\{n_{k}: k \in N\right\}$ such that $n_{1}<n_{2}<\cdots$ and for $k \in N$

$$
J\left(z_{n_{k}}\right) \subset U_{k} .
$$


Then $f\left(z_{n_{k}}\right) \rightarrow y$. In fact, otherwise there exists an open set $V \ni y$ in $Y$ such that $\left\{k: f\left(z_{n_{k}}\right) \notin V\right\}$ is infinite. It follows from $f^{-1}(y) \subset f^{-1}(V)$ and Lemma 2 that there exist $T_{1}, T_{2}, \ldots, T_{m} \in \mathscr{S}$ such that

$$
X \backslash f^{-1}(V) \subset T_{1} \cup T_{2} \cup \cdots \cup T_{m} \subset X \backslash f^{-1}(y) .
$$

Since $\left\{k: f\left(z_{n_{k}}\right) \notin V\right\}$ is infinite, there exists $T_{i}$ such that $\left\{k: z_{n_{k}} \in T_{i}\right\}$ is infinite. Thus (4) implies that

$$
\bigcap\left\{J\left(z_{n_{k}}\right): z_{n_{k}} \in T_{i}\right\} \subset \bigcap\left\{U_{k}: z_{n_{k}} \in T_{i}\right\}=Z .
$$

Hence it follows from (3) and (5) that

$$
\begin{aligned}
& T_{i} \cap \bigcap\left\{J\left(z_{n_{k}}\right): z_{n_{k}} \in T_{i}\right\} \\
& \quad=T_{i} \cap Z \cap \bigcap\left\{J\left(z_{n_{k}}\right): z_{n_{k}} \in T_{i}\right\} \subset T_{i} \cap f^{-1}(y)=\varnothing .
\end{aligned}
$$

On the other hand, the family

$$
\left\{T_{i}\right\} \cup\left\{J\left(z_{n_{k}}\right): z_{n_{k}} \in T_{i}\right\}
$$

is a linked subfamily of $\mathscr{S}$. Hence,

$$
T_{i} \cap \bigcap\left\{J\left(z_{n_{k}}\right): z_{n_{k}} \in T_{i}\right\} \neq \varnothing
$$

since $\mathscr{S}$ is binary (this is the only point in the proof where we use the fact that $\mathscr{S}$ is binary). Now a contradiction occurs.

Remark. For a nonisolated point $y \in Y$ let

$$
t(y)=\min \{|A|: A \subset Y \backslash\{y\} \text { and } \bar{A} \ni y\} .
$$

In Theorem we proved that in certain spaces $Y$ if $t(y)$ is countable, then $y$ is the limit of a nontrivial sequence in $Y$. In fact, it is not difficult to extend the result to a general case. We call $Z \subset X$ to be a $G_{\mu}$-set if $Z=\bigcap\left\{U_{\xi}: \xi<\mu\right\}$ for a decreasing open family $\left\{U_{\xi}: \xi<\mu\right\}$. Then we have

Let $Y$ be a continuous image of a closed $G_{\mu}$-set of a supercompact space and $y \in Y$ a nonisolated point. If $\mu \leq \operatorname{cf}(t(y))$, then $y$ is the limit of a nontrivial. $\alpha$-sequence in $Y$ for some limit ordinal $\alpha \leq t(y)$.

From this statement the following corollary is obtained.

Corollary. If $Y$ is a continuous image of a supercompact space, then every nonisolated point in $Y$ is the limit of a nontrivial linear net.

\section{ACKNOWLEDGMENT}

The author is indebted to Professor Katsuya Eda for simplifying the original proof.

\section{REFERENCES}

1. M. G. Bell, Supercompactness of compactification and hyperspaces, Trans. Amer. Math. Soc. 281 (1984), 717-724.

2. $\frac{1}{775}$ Not all dyadic spaces are supercompact, Comment. Math. Univ. Carolin. 31 (1990), 775-779. 
3. __ A first countable supercompact Hausdoff space with a closed $G_{\delta}$ non-supercompact subspace, Colloq. Math. 43 (1980), 233-241.

4. W. Bula, J. Nikiel, H. M. Tuncali, and E. D. Tymchatyn, Continuous images of ordered compacta are regular supercompact, Topology Appl. 45 (1992), 203-221.

5. E. K. van Douwen, Special bases for compact metrizable spaces, Fund. Math. 61 (1981), 201-209.

6. E. K. van Douwen and J. van Mill, Supercompact spaces, Topology Appl. 13 (1982), 21-32.

7. J. de Groot, Supercompactness and superextension, Contribution to Ext. Theory Top. Struct. Symposium (Berlin), Dentyscher Verlaae der Wissenchafen, Berlin, 1969, pp. 89-90.

8. J. van Mill, Supercompactness and Wallman spaces, Math. Centre Tract, vol. 85, NorthHolland, Amsterdam, 1977.

9. J. van Mill and C. F. Mills, Closed $G_{\delta}$ subset of supercompact Hausdorff spaces, Indag. Math. (N.S.) 41 (1979), 155-162.

10. (1979), 241-247.

11. C. F. Mills, $A$ simpler proof that compact metric spaces are supercompact, Proc. Amer. Math. Soc. 73 (1979), 388-390.

Institute of Mathematics, University of Tsukuba, Ibaraki, Tsukuba 305, Japan

Current address: Department of Mathematics, Shaanxi Normal University, Xi'an, 710062, People's Republic of China 